\title{
Psychometric Properties of Persian Version of the Rheumatoid Arthritis Quality of Life scale
}

\author{
Mina Vesal ${ }^{*}$, Maryam Ghaemi², Mohammad Ali Nazarinia ${ }^{3}$ \\ ${ }^{1}$ Faculty of Education and Psychology, Isfahan University, Isfahan, Iran \\ ${ }^{2}$ Faculty of Education and Psychology, Shiraz University, Shiraz, Iran \\ ${ }^{3}$ Geriatric Research Center, Shiraz University of Medical Sciences, Shiraz, Iran
}

\section{ABSTRACT}

Introduction: Rheumatoid arthritis (RA) is a chronic inflammatory disease characterized by a pattern of destruction of bones and joints. Quality of life is one of the important psychological factors in the field of public health. The purpose of this study was to determine the psychometric indices of the Persian version of the Rheumatoid Arthritis Quality of Life questionnaire (RAQoL). Materials and Methods: In this descriptive study, 93 patients with RA referred to Hafez hospital Rheumatology Clinic of Shiraz University of Medical Sciences were selected through a purposive sampling method and they responded to the RAQoL and the Depression Anxiety Stress Scales (DASS-21). Convergent validity was calculated by measuring the correlation between the quality of life and depression, anxiety, and tension. Results: The reliability of the scale was confirmed by an Alpha factor of 0.88 , indicating a high internal consistency. In assessing convergent validity, the lowest quality of life was associated with depression symptoms $(\mathrm{R}=-0.75)$ stress $(\mathrm{R}=-0.67)$ and anxiety $(\mathrm{R}=$ -0.59) at the 0.01 level of significance. Conclusion: Our findings indicate that the Persian version of the RAQOL questionnaire has reliability and validity and is sensitive to changes in RA in clinical situations. Furthermore, it can be used as a useful self-assessment tool for research and counseling.

\section{Key words:}

1. Quality of Life

2. Arthritis, Rheumatoid

3. Patients

*Corresponding Author: Mina Vesal

E-mail:mvesal@edu.ui.ac.ir 


\title{
ويثزَى هاى روانســنجى نسخةٌ فارسى يرسشنامةُ كيفيّت زندَّى روماتيسم مفصلى
}

\author{
مينا وصال"'، مريم قائمى '، محمدعلى نظرى نياّ \\ 'دانشكده علوم تربيتى و روانشناسى، دانشعاه اصفهان، اصفهان، ايران \\ rانشكده علوم تربيتى و روانشناسى، دانشعاه شيراز، شيراز، ايران \\ 'مركز تحقيقات سالمندى، دانشكاه علوم يزشكى شيراز، شيراز، ايران
}

\section{اطلاعات مقاله:}

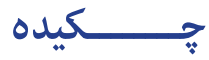

مقدمه: روماتيسم مفصلى يك بيمارى التهابى مزمن است كه با الخوى تخريب استخوانها و مفاصل

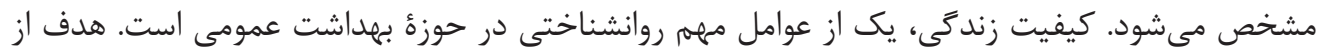

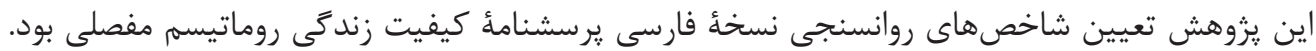

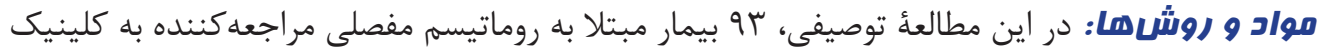

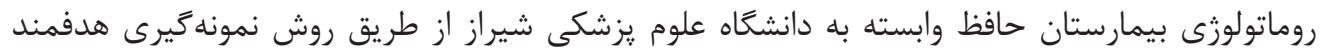

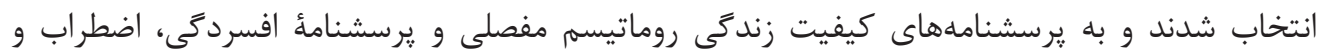
تنيد

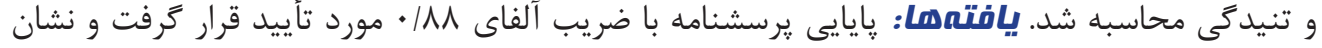

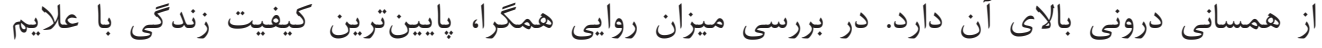

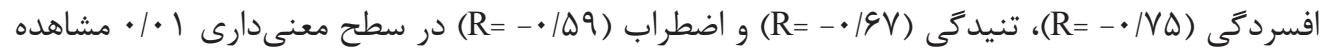

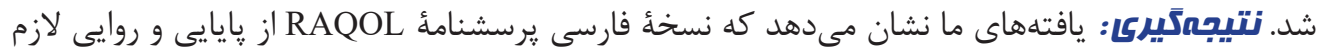

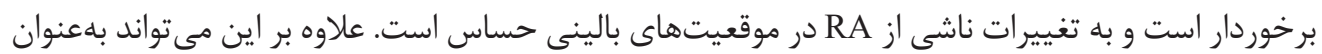

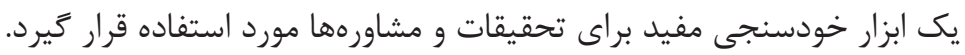

كليد وازمها:

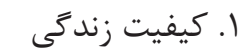

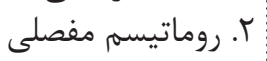

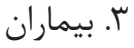

* نويسنده مسئول: مينا وصال آدرس الكترونيكى: mvesal@edu.ui.ac.ir 


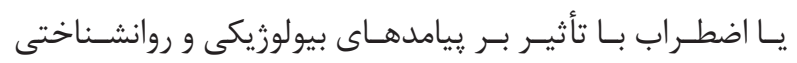

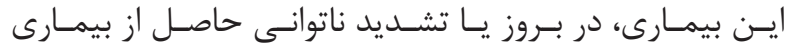

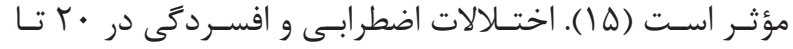

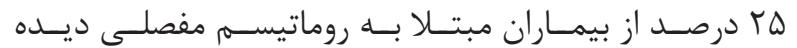

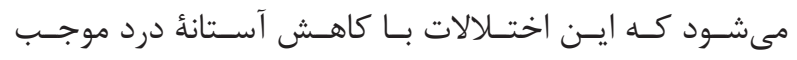

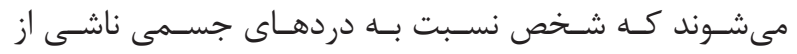

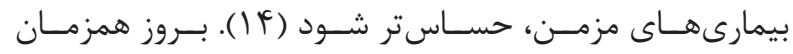

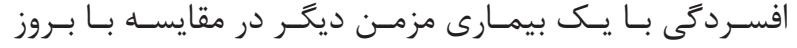

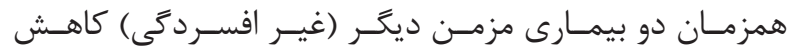

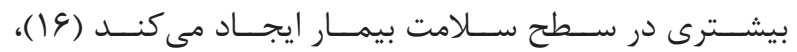

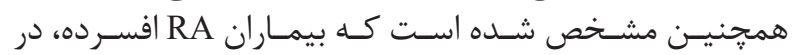

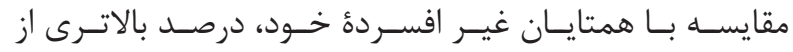

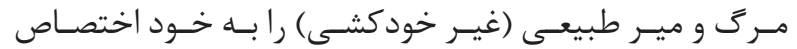

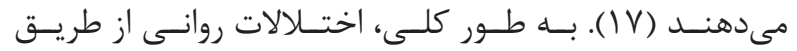

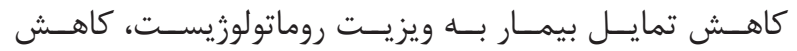

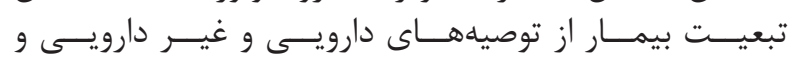

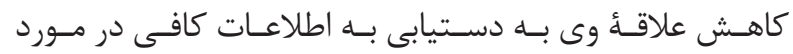

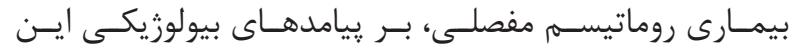

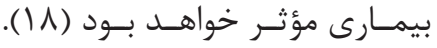

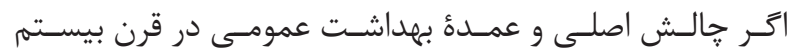

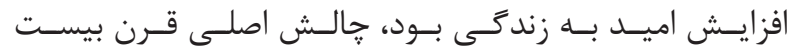

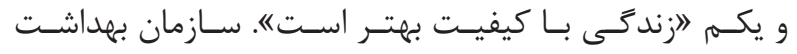

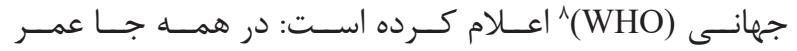

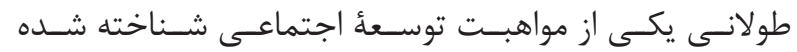

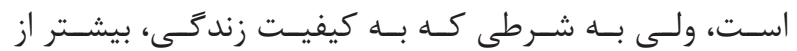

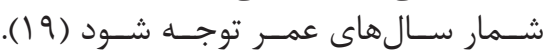

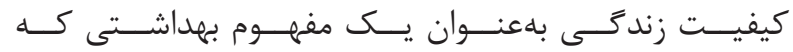

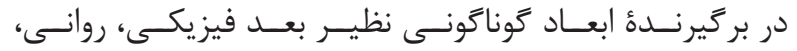

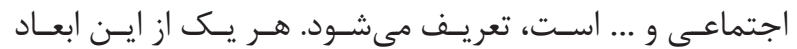

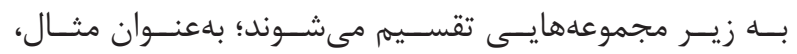

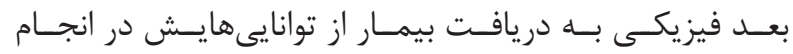

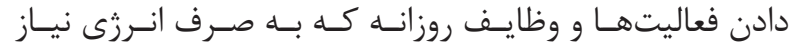

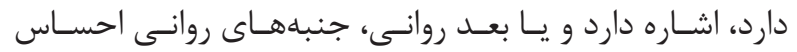

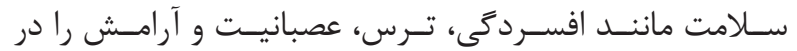

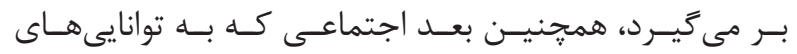

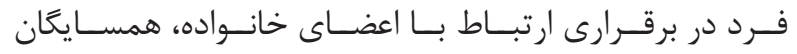

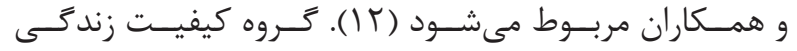

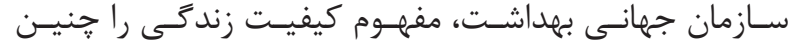

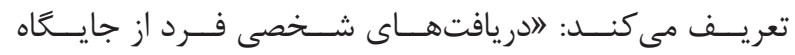

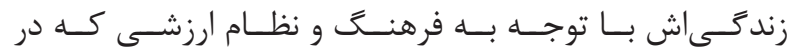

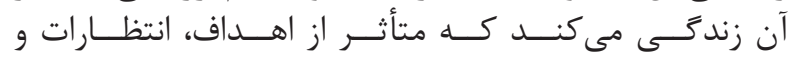

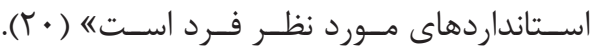

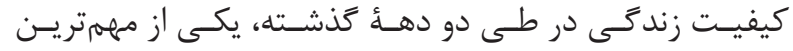

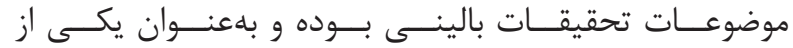

${ }^{1}$ Rheumatoid arthritis

${ }^{2}$ Autoimmune, inflammatory and multifactorial disease

${ }^{3}$ Unknown etiology

${ }^{4}$ Synovium

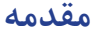

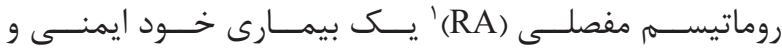

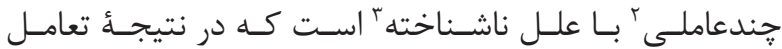

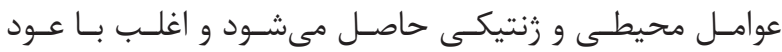

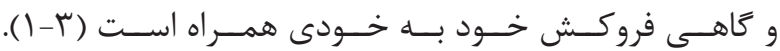

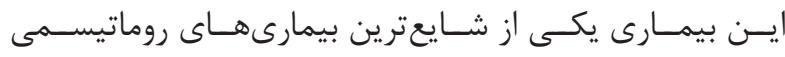

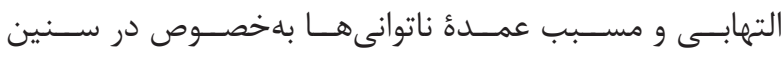

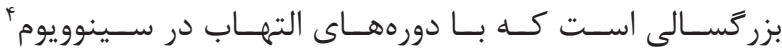

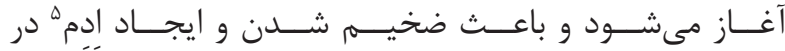

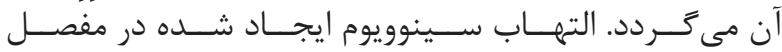

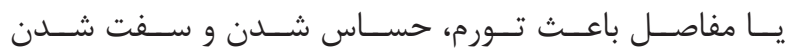

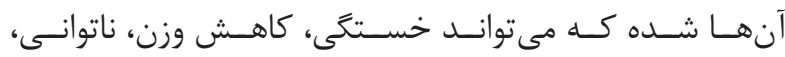

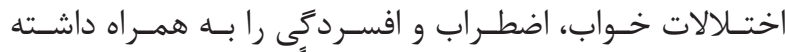

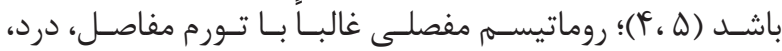

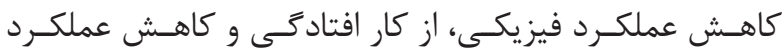

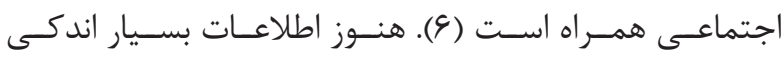

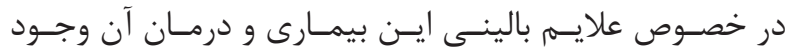

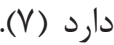

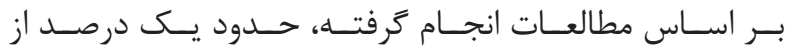

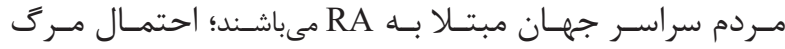

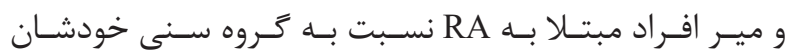

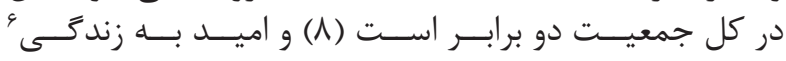

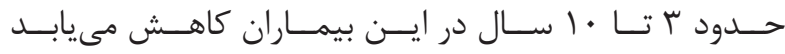

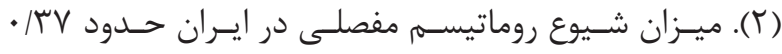

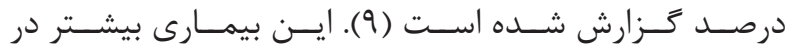

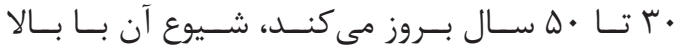

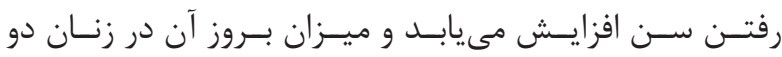

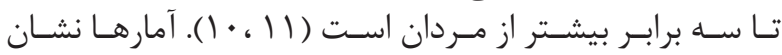

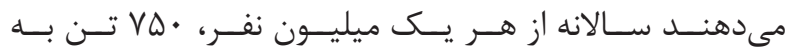

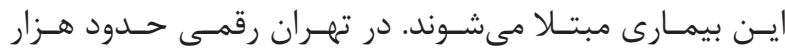

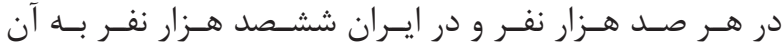

مبتـلا هسـتـند (I) (I).

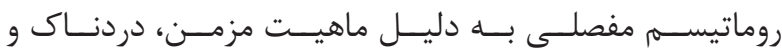

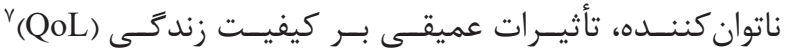

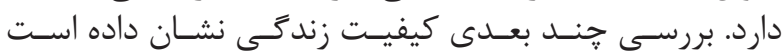

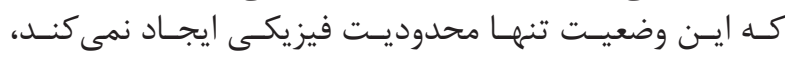

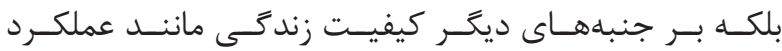

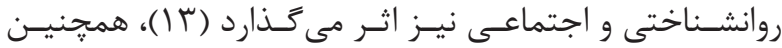

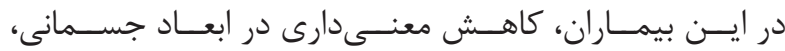

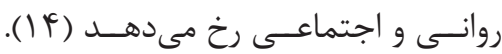

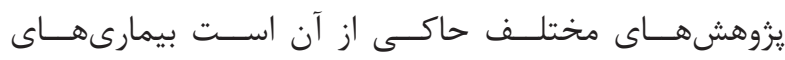

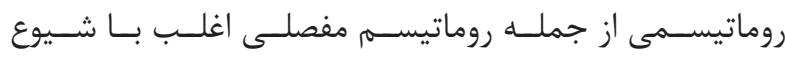

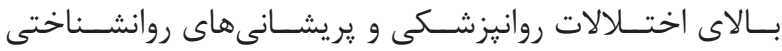

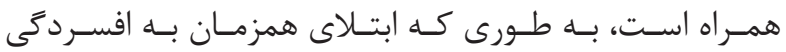

${ }^{5}$ Edema

${ }^{6}$ The life expectancy

${ }^{7}$ Quality of life

${ }^{8}$ World health organization 


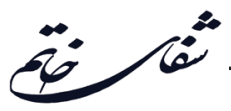

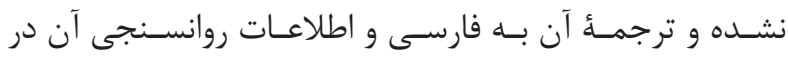

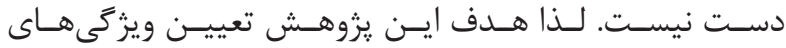

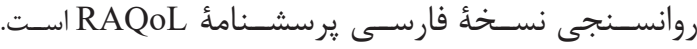

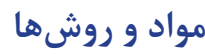

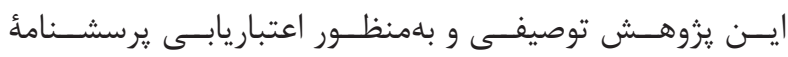

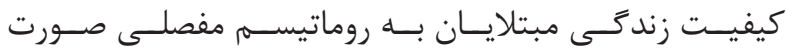

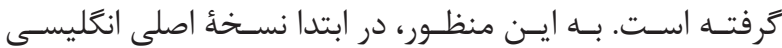

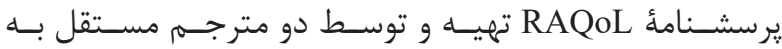

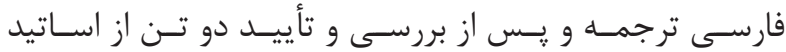

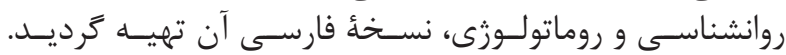

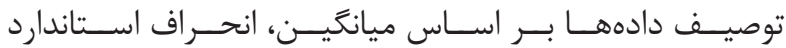

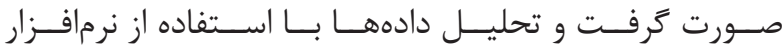
از SPS-23

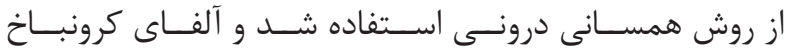

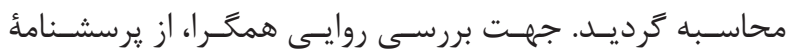

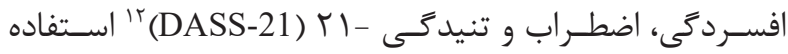

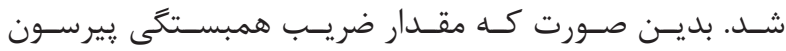

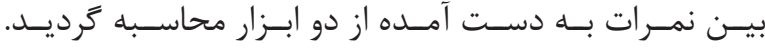

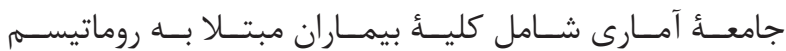

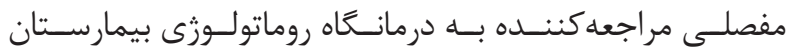

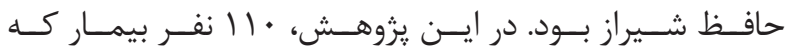

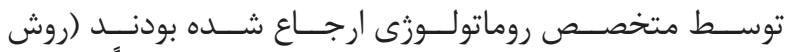

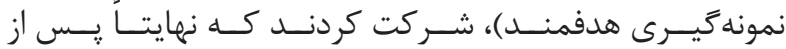

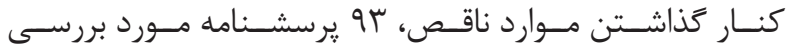

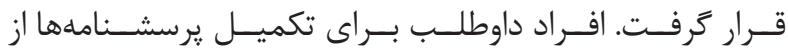

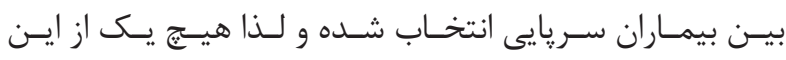

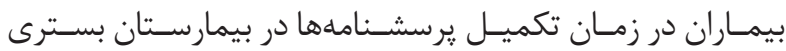

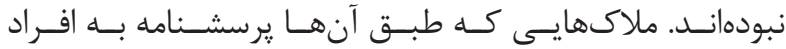

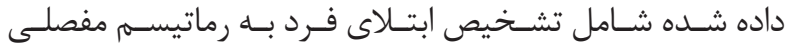

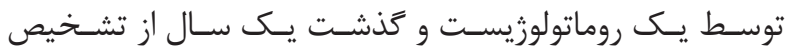

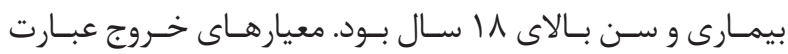

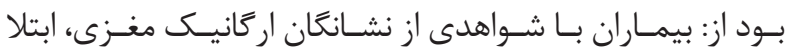

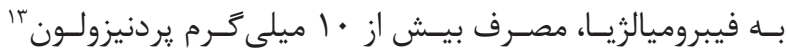

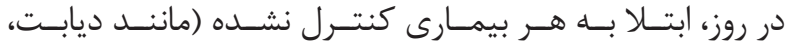

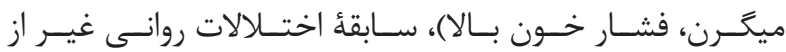

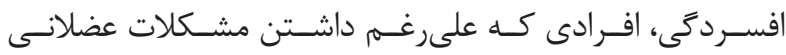

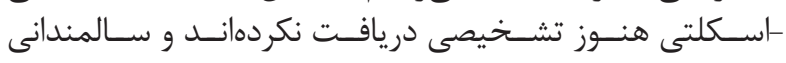

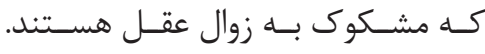

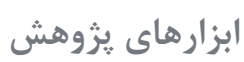

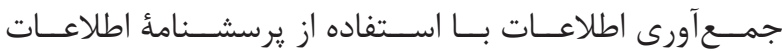

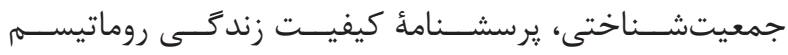

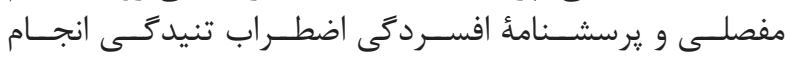

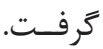

${ }^{9}$ Disease-specific measures

${ }^{10}$ Needs-based QOL model

${ }^{11}$ De Jon

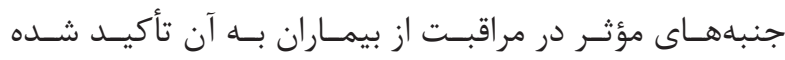

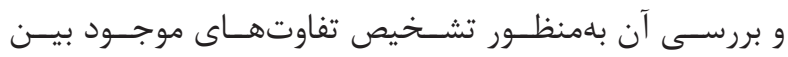

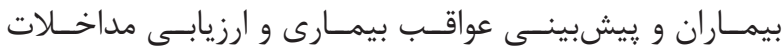

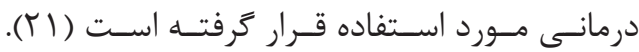

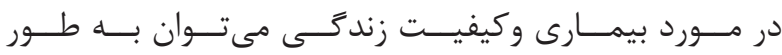

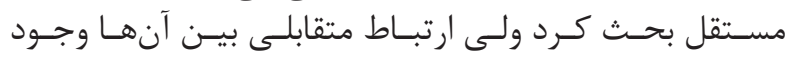

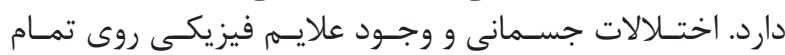

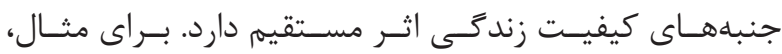

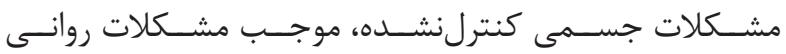

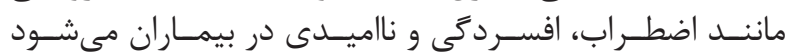

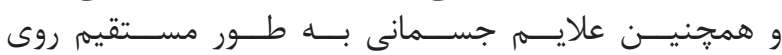

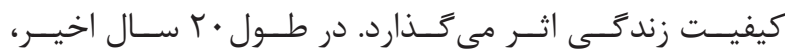

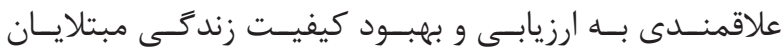

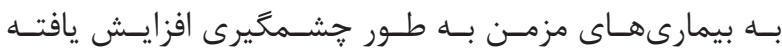

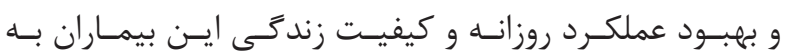

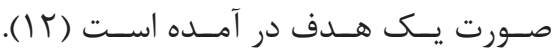

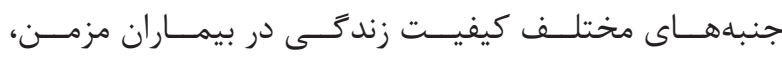

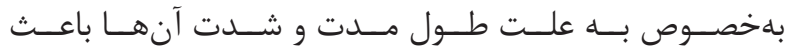

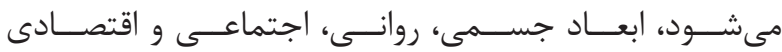

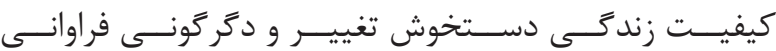

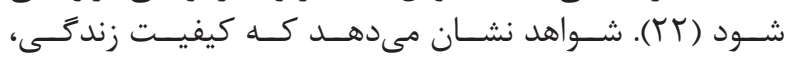

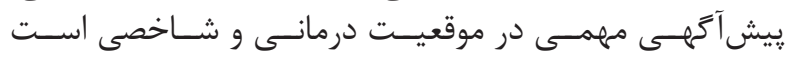

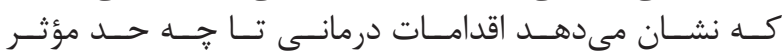

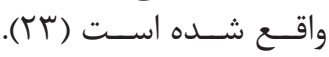

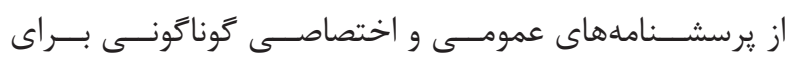

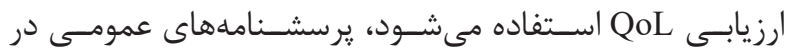

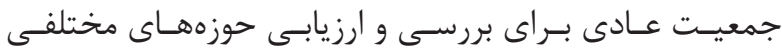

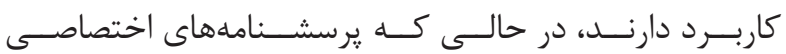

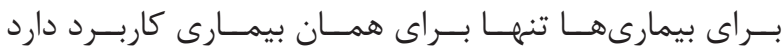

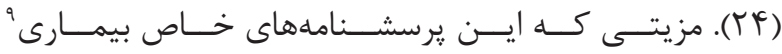

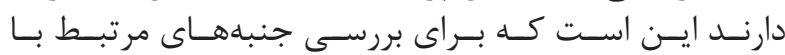

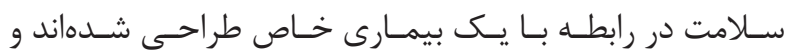

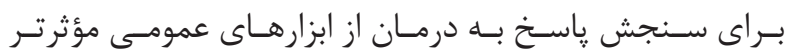

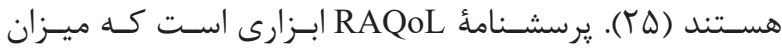

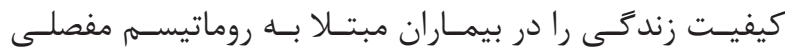

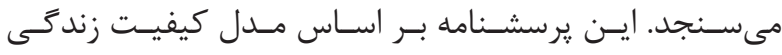

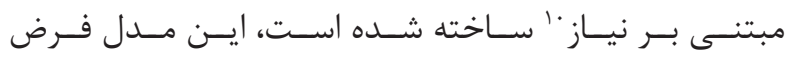

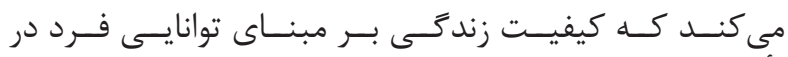

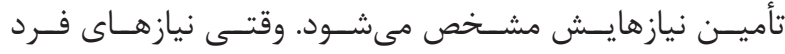

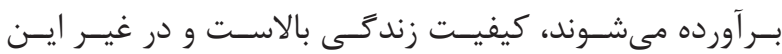

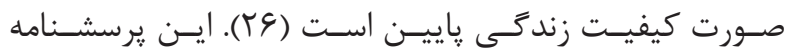

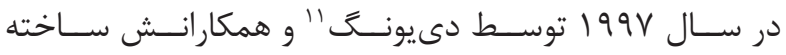

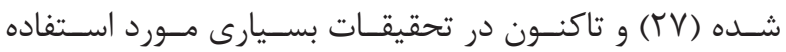

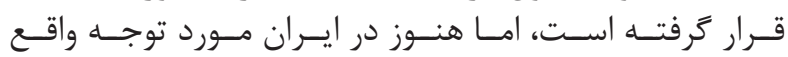

${ }^{12}$ The depression anxiety stress scales

${ }^{13}$ Prednisolone 


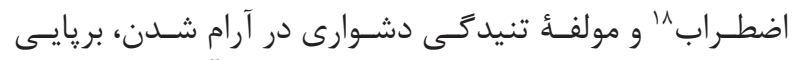

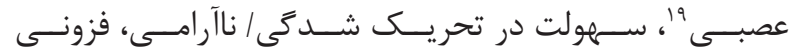

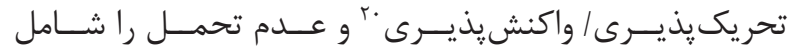

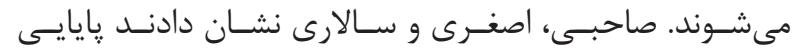

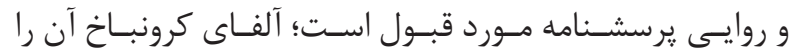

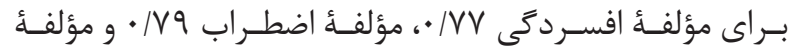

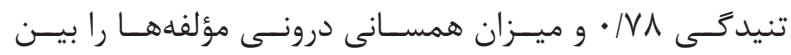

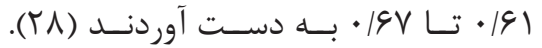

يافتهها

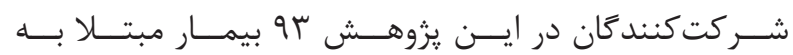

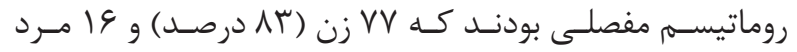

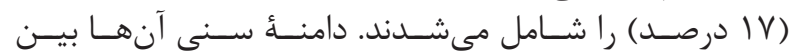

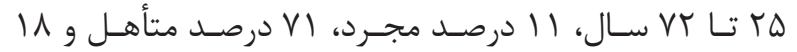

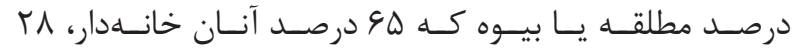

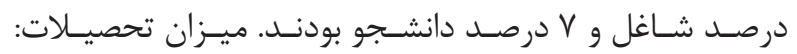

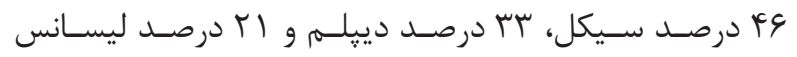

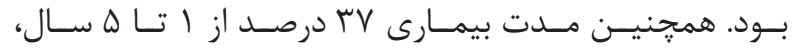

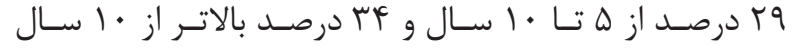

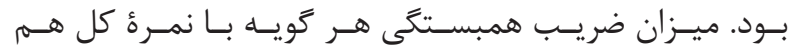

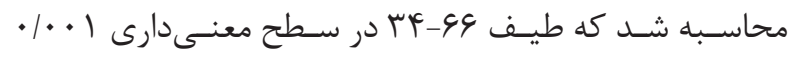

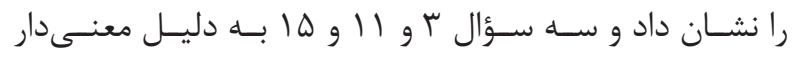

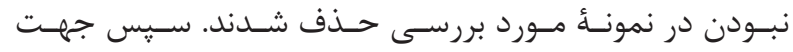

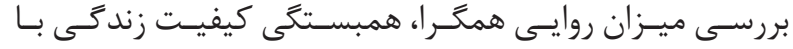

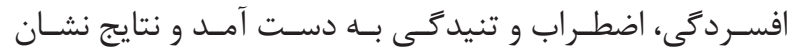

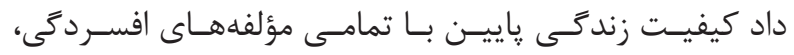

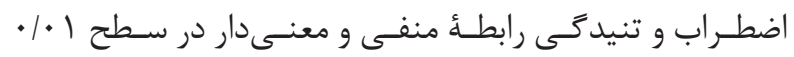

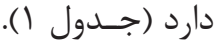

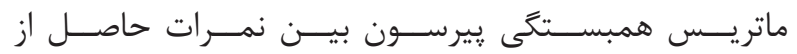

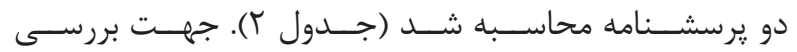

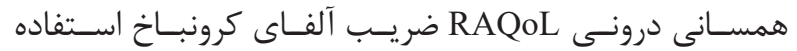

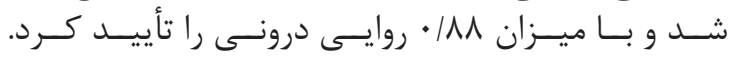

\section{برسشنامةُ اطلاعات جمعيتشناختى}

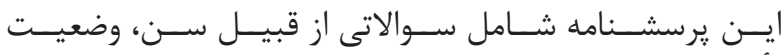

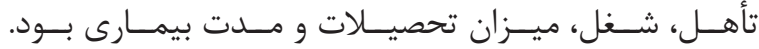
يرسشنامة كيفيت زندگى روماتيسم مفصلى

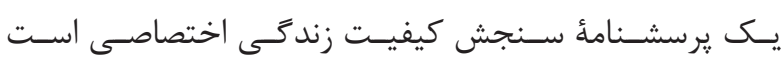

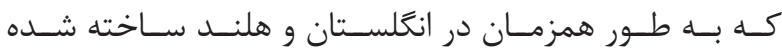

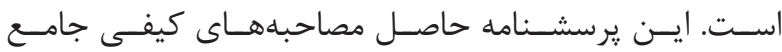

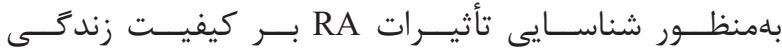

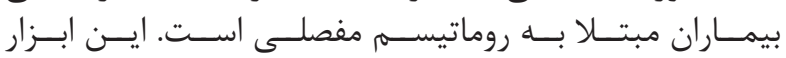

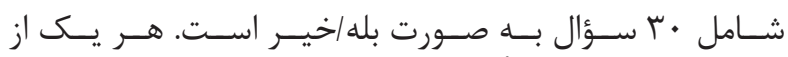

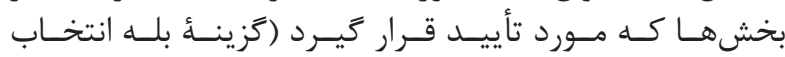

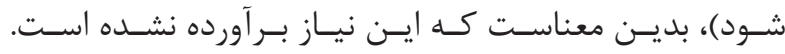

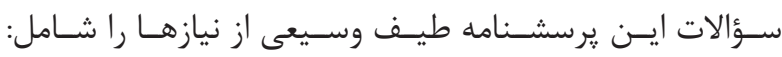

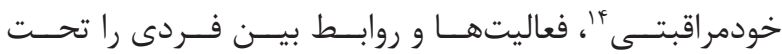

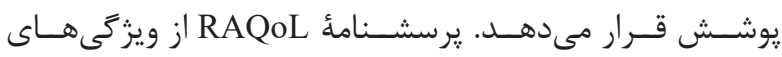

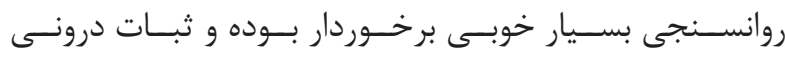

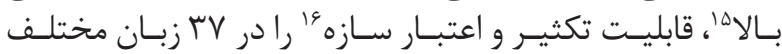

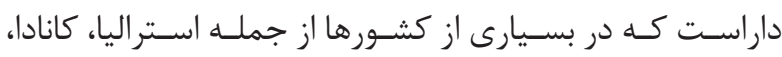

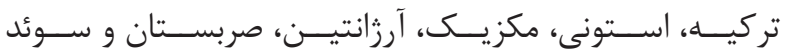

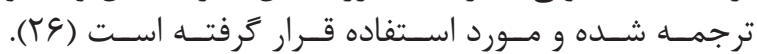

\section{يرسشنامةٔ افسردَى اضطراب تنيدَى}

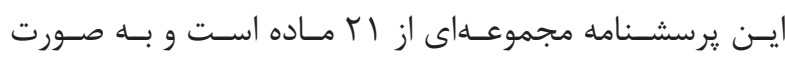

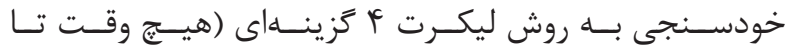

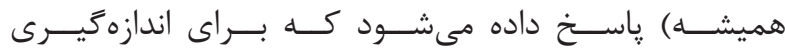

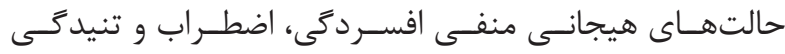

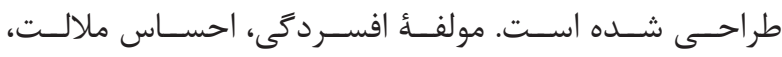

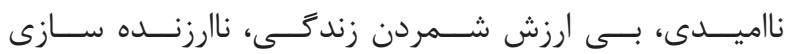

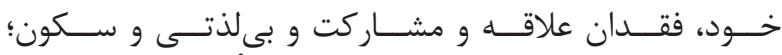

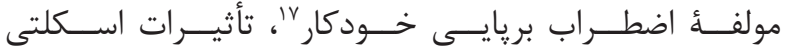

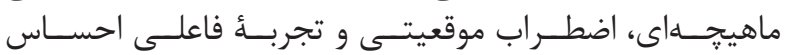

جدول ا- آمارههاى توصيفى مربوط به متغيرهاى يُروهش.

\begin{tabular}{|c|c|c|c|c|}
\hline بالاترين نمره & يايينترين نمره & انحراف استاندارد & ميانكين & مؤلفه \\
\hline rq & f & $9 / 9$ & $\mid f / V$ & كيفيت زندگى \\
\hline ra & $\wedge$ & $f / r$ & $10 / V$ & افسردتى \\
\hline rt & V & $r / l$ & r & اضطراب \\
\hline re & 11 & $r / \Delta$ & $11 / K$ & تنيدگى \\
\hline
\end{tabular}

\begin{tabular}{|c|c|c|c|c|}
\hline تنيدكى & اضطراب & افسردكى & كيفيت زندكى & مؤلفهها \\
\hline - & - & - & 1 & كيفيت زندكى \\
\hline- & - & 1 & $-\cdot / V \omega^{* 3}$ & افسردكى \\
\hline - & 1 & $\cdot 19\}^{403}$ & $-\cdot / \Delta q^{*}$ & اضطراب \\
\hline 1 & $\cdot / \Delta \omega^{*}$ & $\cdot / V r^{* * 3 *}$ & $-\cdot 19 V^{3 * 40}$ & تنيدكى \\
\hline
\end{tabular}

${ }^{14}$ Self-care

${ }^{15}$ High internal consistency

${ }^{16}$ Reproducibility and construct validity

${ }^{17}$ Autonomic arousal
${ }^{18}$ Subjective experience of anxious affect

${ }^{19}$ Nervous arousal

${ }^{20}$ Irritable/ over-reactive 
بــا افزايـش اســفاده از خدمـات بهداشـتى و افزايـش ميـزان

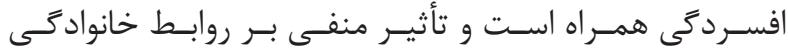

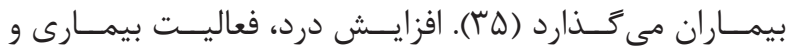

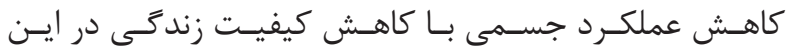

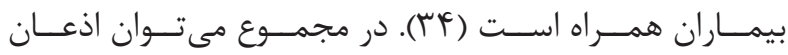

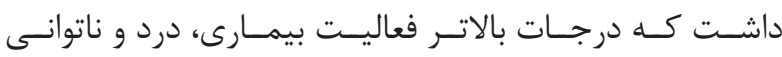

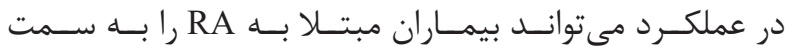

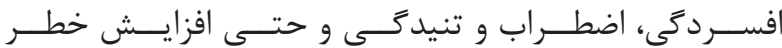

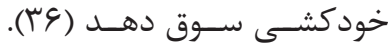

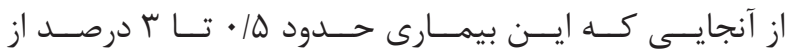

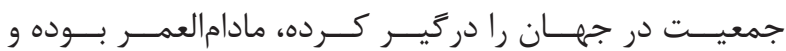

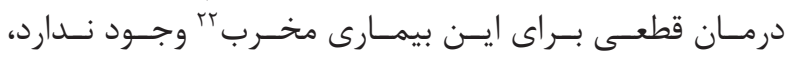

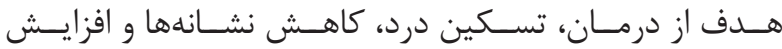

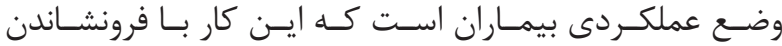

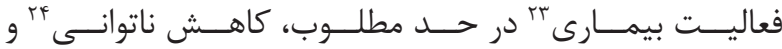

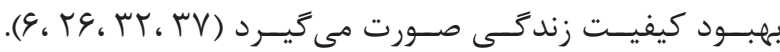

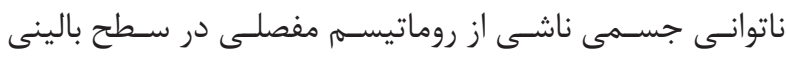

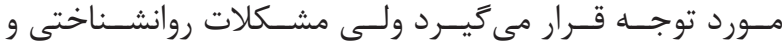

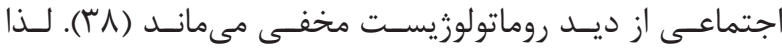

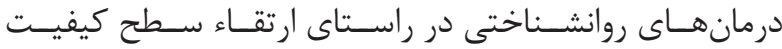

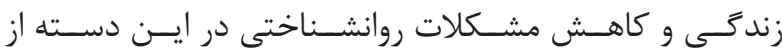

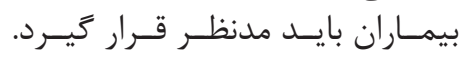

$$
\text { تشكر و قدردانى }
$$

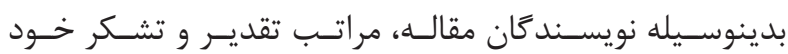

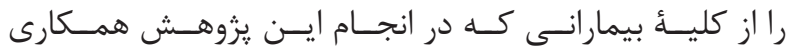

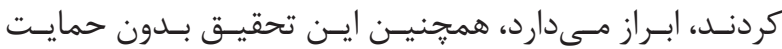

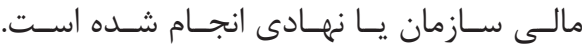

بحث و نتيجه كيرى

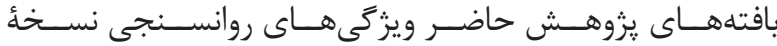

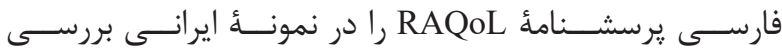

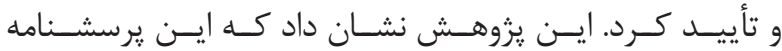

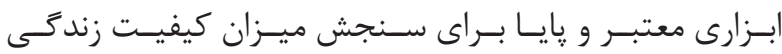

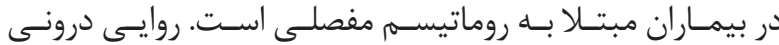

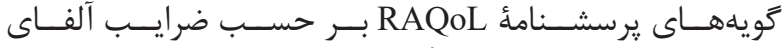

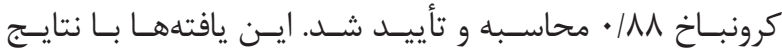

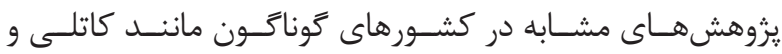

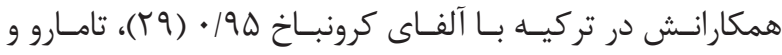

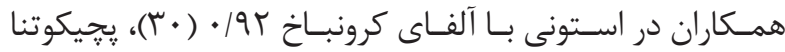

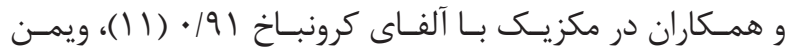

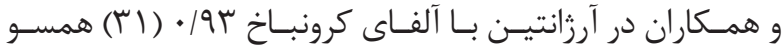

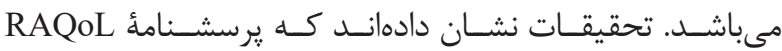

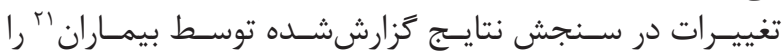

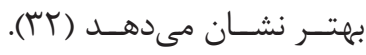

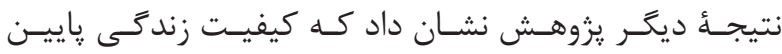

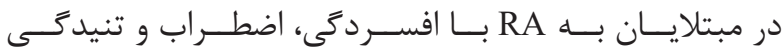

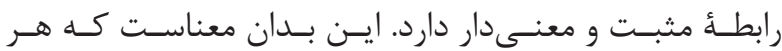

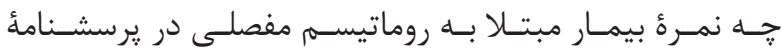

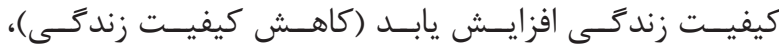

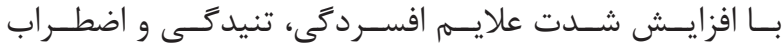

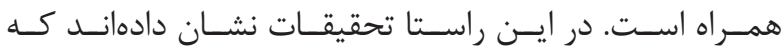

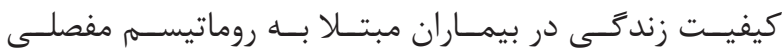

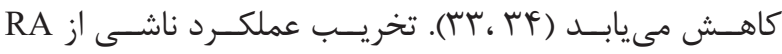

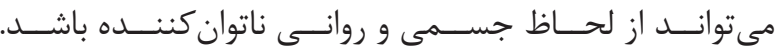

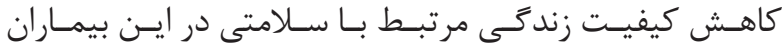

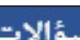

\begin{tabular}{|c|c|c|c|}
\hline \multicolumn{3}{|r|}{ سؤالات } & رديف \\
\hline خحير & بلى & بايد زودتر از آتجه مايلم به رختخواب بروم. & 1 \\
\hline حمير & بلى & از اينكة سايرين مرا لمس كيند، مى ترسمه. & r \\
\hline خمير & بلى & نيدا كردن كقشى راحتى كه دوست دارم، برايهم دشوار است. & r \\
\hline خدير & بلى & به خاطر شرايطهم از حضور در مكانهاى شلوغ امتناع مىكثمه. & F \\
\hline خير & بلى & لباد بوشيدن برايهم قشوار است. & $\Delta$ \\
\hline خير & بلى & فهميدم كه بياده خريد كردن، برايهم سخت است. & 8 \\
\hline مير & 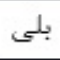 & كارهاى خماته وقت زيادى از من مىكيرد. & $\mathrm{r}$ \\
\hline خ خير & بلى & كاهمى اوقات استفاده از سرويسى بهداشتى برايمم دشوار است. & $\wedge$ \\
\hline خ خير & بلى & اغلب احسادي ناكامى ميىكثم. & 9 \\
\hline خير & 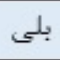 & در حين التجام كار مجبورم براى استراحت، كارم را متوقف كـهم. & 1. \\
\hline مير & بلى & استقاده از كارد و هنكال برايمه مشكل است. & 川 \\
\hline خير & 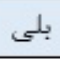 & متوجنه شدم كه تمركز كردن برايهم دشوار است. & it \\
\hline مير & بلى & بعضى وقتها فقط مي خخواهم تنهايهم بكذارتد. & Ir \\
\hline مخير & بلى & دريافتم كه راه رقتن طولاثى برايمم سخت است. & if \\
\hline
\end{tabular}

${ }^{21}$ Patient-reported outcome measure

${ }^{22}$ Destructive disease
${ }^{23}$ Disease activity

${ }^{24}$ Disability 


\begin{tabular}{|c|c|c|c|}
\hline \multicolumn{4}{|c|}{ 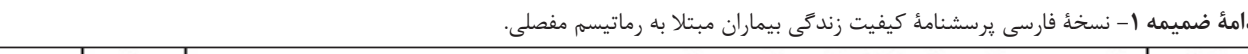 } \\
\hline خحير & بلى الى & از دست دادن با ديكران اجتناب مىكنهم. & 10 \\
\hline خير & 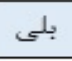 & اغلب افسرده ميىشوم. & 19 \\
\hline خير & 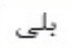 & تمى تواتم به فعاليّت هاى خحاتوادكى و دوستاته بنييوتدم. & iv \\
\hline خدير & بلى & دريافتهم در دوثى كرفتن الحمام كردن مشكل دارم. & 11 \\
\hline حير & بلى & به خخاطر شرايطهم: كاهى وقتها شديدأ كريه ميى كنمه. & 19 \\
\hline خير & 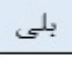 & شرايطهم، مرا در رفتن به مكان هايَى كه مىتواتهم برومه محدود مى كند. & r. \\
\hline خير & 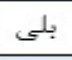 & از التجام هركارى الحساس خستكى مي كنيم- & ו \\
\hline خير & بلى & احساس مى كثمر به ديكران وايسته هستمه. & rt \\
\hline حير & بلى & شرايطهم همواره در ذهنميم است. & rr \\
\hline خير & 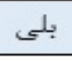 & اغلب از دست خودم عصداتى هستمه & rF \\
\hline خدير & بلى & 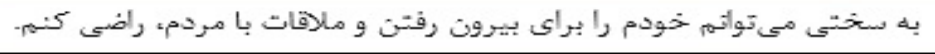 & ro \\
\hline خ خير & بلى & شببها بد مي خوابجم. & rs \\
\hline خدير & 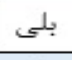 & فهميدهام كه مراقبت از تزديكان برايهم دشوار است. & rr \\
\hline حخير & بلى & احساسى مى ك-هم تمى تواتم شرايطهم را كنترل ك-هم- & $r \lambda$ \\
\hline حمير & بلى & از برخوردهاى فيزيكى اجتتاب مىكسهم. & rq \\
\hline خدير & بلى الى & در يوشيدن لباسى با محدوديّت مواجه هستمب. & r. \\
\hline
\end{tabular}

1. Lawrence RC, Felson DT, Helmick CG, Arnold LM, Choi H, Deyo RA, et al. Estimates of the prevalence of arthritis and other rheumatic conditions in the United States: Part II. Arthritis Rheum. 2008; 58(1): 26-35.

2. Tobón GJ, Youinou P, Saraux A. The environment, geoepidemiology, and autoimmune disease: Rheumatoid arthritis. J Autoimmun. 2010; 35(1): 10-4.

3. Kanecki K, Tyszko P, Wisłowska M, ŁyczkowskaPiotrowska J. Preliminary report on a study of healthrelated quality of life in patients with rheumatoid arthritis. Rheumatol Int. 2013; 33(2): 429-34.

4. Chen S, Wang H. The relationship between physical function, knowledge of disease, social support and selfcare behavior in patients with rheumatoid arthritis. J Nurs Res. 2007; 15(3): 83-92.

5. Goes ACJ, Reis L, Silva MBG, Kahlow B, Skare TL. Rheumatoid arthritis and sleep quality. Revista Brasileira de Reumatologia. 2017; 57(4): 294-8.

6. Cox SR, McWilliams L, Massy-Westropp N, Meads DM, McKenna SP, Proudman S. Adaptation of the RA QoL for use in Australia. Rheumatology International. 2007; 27: 661-6.

7. Michaud K, Wolfe F. Comorbidities in rheumatoid arthritis. Best Practice \& Research Clinical Rheumatology. 2007; 21(5): 885-906.

8. Morowatisharifabad M, Nadrian H, Soleimani Salehabadi H, Mazloomy Mahmoodabad SMA. The Relationship between Predisposing Factors and Self- care Behaviors among Patients with Rheumatoid Arthritis. Journal of Hayat. 2009; 15(3): 39-51.

9. Davatchi F, Sandoughi M, Moghimi N, Jamshidi A, Tehrani Banihashemi A, Zakeri Z, et al. Epidemiology of rheumatic diseases in Iran from analysis of four COPCORD studies. International Journal of Rheumatic Diseases. 2016; 19(11): 1056-62.

10. Pakpour AH, Zeidi IM, Hashemi F, Saffari M, Burri A. Health-related quality of life in young adult patients with rheumatoid arthritis in Iran: reliability and validity of the Persian translation of the PedsQL ${ }^{\text {TM }} 4.0$ generic core scales young adult version. Clinical Rheumatology. 2013; 32(1): 15-22.

11. Pacheco-Tena C, Reyes-Cordero G, Mckenna SP, Ríos-Barrera VA. Adaptación y validación del Rheumatoid Arthritis Quality of Life Scale (RAQoL) al español de México. Reumatologia Clinica. 2011; 7(2): 98-103.

12. Razavian F, Abbasi M, Kazemnejad A. The relationship between depression and the quality of life in patients with rheumatoid arthritis. Daneshvar Medicine. 2009; 16(80): 27-34.

13. King IM. Quality of life and goal attainment. Nursing Science Quarterly. 1994; 7(1): 29-32.

14. Jain A, Bhadauria D. Evaluation of efficacy of fluoxetine in the management of major depression and arthritis in patients of Rheumatoid Arthritis. Indian Journal of Rheumatology. 2013; 8(4): 165-9. 


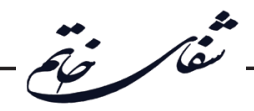

15. M. V, M. N. Prediction of depression and sleep quality based on thought rumination and its components (inhibition and reflection) in patients with Rheumatoid arthritis. Journal of Thought \& Behavior in Clinical Psychology. 2016; 9(41): 47-56.

16.Mardani Hamouleh M, Shahraki Vahed A, Moshtagh Eshgh Z. Assessment of the relationship between mental health and job stress among nurses. Pars Journal of Medical Sciences. 2010; 8(2): 35-40.

17. Lawrence A, Aggarwal A. Depression in rheumatoid arthritis: An under-recognized comorbidity. Indian Journal of Rheumatology. 2013; 4(8): 153-4.

18. DiMatteo MR, Lepper HS, Croghan TW. Depression is a risk factor for noncompliance with medical treatment: meta-analysis of the effects of anxiety and depression on patient adherence. Archives of Internal Medicine. 2000; 160(14): 2101-7.

19. Sajadi H, Biglarian A. The quality of life of the elderly woman in the Kahrizak charity nursing home. Payesh. 2004; 5(2): 105-8.

20. Karimlou M, Salehi M, Zayeri F, Massah O, Hatami A, Moosavy Khatat M. Developing the persian version of the world health organization quality of life 100- ques tionnaire. Archives of Rehabilitation. 2011; 11(4): 73-82.

21. Gill TM, Feinstein AR. A critical appraisal of the quality of quality-of-life measurements. Jama. 1994; 272(8): 619-26.

22. Omidi A, Momeni J, Raygan F, Akbari H, Talighi E. The effectiveness of mindfulness-based stress reduction program on quality of life among patients with cardiovascular diseases. Journal of Thought \& Behavior in Clinical Psychology. 2017; 10(42): 7-16.

23. Izadi S, Neamat Tavousi M. The effectiveness of mindfulness therapy in quality of life among patients with MS. Journal of Thought \& Behavior in Clinical Psychology. 2017; 11(42): 37-46.

24. Garip Y, Eser F, Bodur H. Health-related quality of life in rheumatoid arthritis: comparison of RAQoL with other scales in terms of disease activity, severity of pain, and functional status. Rheumatology International. 2011; 31(6): 769-72.

25. Haroon N, Aggarwal A, Lawrence A, Agarwal V, Misra R. Impact of rheumatoid arthritis on quality of life. Modern Rheumatology. 2007; 17(4): 290-5.

26 .Zlatkovic-Svenda M, Rouse M, Radak-Perovic M, Stojanovic R, Vujasinovic-Stupar N, Lazovic-Popovic $\mathrm{B}$, et al. Adaptation and validation of the Rheumatoid Arthritis Quality of Life (RAQoL) questionnaire for use in Serbia. Rheumatology International. 2017; 37(4): 641-6.
27. De Jong Z, Van der Heijde D, McKenna S, Whalley D. The reliability and construct validity of the RAQoL: a rheumatoid arthritis-specific quality of life instrument. Rheumatology. 1997; 36(8): 878-83.

28. Sahebi A, Asghari M, Salari R. Validation of depression anxiety and stress scale (dass-21) for an iranian population. Iranian Psychologists. 2005; 1(14): 36-54.

29. Kutlay S, Küçükdeveci AA, Gönül D, Tennant A. Adaptation and validation of the Turkish version of the Rheumatoid Arthritis Quality of Life Scale. Rheumatology International. 2003; 23(1): 21-6.

30. Tammaru M, McKenna SP, Meads DM, Maimets K, Hansen E. Adaptation of the rheumatoid arthritis quality of life scale for Estonia. Rheumatology International. 2006; 26(7): 655-62.

31. Waimann CA, Dal Pra FM, Marengo MF, Schneeberger EE, Gagliardi S, Cocco JAM, et al. Quality of life of patients with rheumatoid arthritis in Argentina: reliability, validity, and sensitivity to change of a Spanish version of the rheumatoid arthritis quality of life questionnaire. Clinical Rheumatology. 2012; 31(7): 1065-71.

32. Wilburn J, McKenna SP, Twiss J, Rouse M, Korkosz M, Jancovic R, et al. Further international adaptation and validation of the Rheumatoid Arthritis Quality of Life (RAQoL) questionnaire. Rheumatology International. 2015; 35(4): 669-75.

33 .Strand V, Singh JA. Improved health-related quality of life with effective disease-modifying antirheumatic drugs: evidence from randomized controlled trials. Am J Manag Care. 2008; 14(4): 234-54.

34. Matcham F, Scott IC, Rayner L, Hotopf M, Kingsley $\mathrm{GH}$, Norton $\mathrm{S}$, et al. The impact of rheumatoid arthritis on quality-of-life assessed using the SF-36: a syststematic review and meta-analysis. Seminars in Arthritis and Rheumatism. 2014; 44(2): 123-30.

35. Gamal RM, Mahran SA, El Fetoh NA, Janbi F. Quality of life assessment in Egyptian rheumatoid arthritis patients: Relation to clinical features and disease activity. The Egyptian Rheumatologist. 2016; 38(2): 65-70.

36. Cunha M, Ribeiro A, André S. Anxiety, depression and stress in patients with rheumatoid arthritis. ProcediaSocial and Behavioral Sciences. 2016; 217: 337-43.

37. Wickrematilake GWG, Wijeratne LS. Assessing the impact of rheumatoid arthritis on quality of life in a group of patients attending a rheumatology clinic in Sri Lanka. Indian Journal of Rheumatology. 2013; 8(1): 14-8.

38. Al-Fadl EMA, Ismail MA, Thabit M, El-Serogy Y. Assessment of health-related quality of life, anxiety and depression in patients with early rheumatoid arthritis. The Egyptian Rheumatologist. 2014; 36(2): 51-6. 